\title{
Predictive factors for endoleaks after thoracic aortic aneurysm endograft repair
}

\author{
Gabriele Piffaretti, MD, ${ }^{\mathrm{a}}$ Giovanni Mariscalco, MD, PhD, ${ }^{\mathrm{b}}$ Chiara Lomazzi, MD, ${ }^{\mathrm{a}}$ Nicola Rivolta, MD, \\ Francesca Riva, MD, ${ }^{\mathrm{a}}$ Matteo Tozzi, MD, ${ }^{\mathrm{a}}$ Gianpaolo Carrafiello, MD, ${ }^{\mathrm{c}}$ Alessandro Bacuzzi, MD, ${ }^{\mathrm{d}}$ \\ Monica Mangini, MD, ${ }^{\mathrm{c}}$ Maciej Banach, MD, PhD, ${ }^{\mathrm{e}}$ and Patrizio Castelli, MD, FACS ${ }^{\mathrm{a}}$
}

\begin{abstract}
Background: Our prospective investigation aimed to determine and analyze the incidence and the determinants of endoleaks after thoracic stent graft.

Methods: Sixty-one patients affected by thoracic aortic aneurysms were treated between January 2000 and March 2008. The study cohort contained 54 men, with a mean age of $63.6 \pm 17.9$ years. The follow-up imaging protocol included chest radiographs and triple-phase computed tomographic angiography performed at 1,4 , and 12 postoperative months and annually thereafter.
\end{abstract}

\begin{abstract}
Results: Median follow-up was 32.4 months (range: 1-96 months). Endoleaks were detected in 9 (14.7\%) patients, of which 7 were type 1 . Five endoleaks were detected at 30 postoperative days, and the other 4 developed with a mean delay of 12 months. Endovascular or hybrid interventions were used to treat the endoleaks. Secondary technical success rate was $100 \%$. Multivariate analysis demonstrated that the diameter of the aneurysmal aorta (odds ratio 1.75, 95\% confidence interval 1.07-2.86) and the coverage of the left subclavian artery (odds ratio $12.05,95 \%$ confidence interval 1.28-113.30) were independently associated with endoleak development. The percentages of patients in whom reinterventions were unnecessary were $94.6 \% \pm 3.0 \%, 88.3 \% \pm$ $4.5 \%$, and $85.4 \% \pm 5.2 \%$, at 1,2 , and 5 years, respectively. The actuarial survival estimates at 1,2 , and 5 years were $85.2 \% \pm 4.6 \%, 78.1 \% \pm 5.4 \%$, and $70.6 \% \pm 6.4 \%$, respectively.
\end{abstract}

Conclusions: The diameter of the aneurysmal aorta and the position of the landing zone are independent predictors of endoleak occurrence after thoracic stent-graft procedures. A careful follow-up program should be considered in patients in whom these indices are unfavorable, because most of the endoleaks may be successfully and promptly treated by additional endovascular procedures.

Endovascular techniques have evolved rapidly and become an accepted alternative to open aortic aneurysm repair. They have also generated new complications including stent-graft (SG) migration and endoleak formation and therefore altered the way patients are followed after repair. ${ }^{1}$ Unlike the minimal imaging required after open surgical repair, patients having endovascular repair of thoracic aortic aneurysms (TEVAR) require lifelong postoperative surveillance imaging. ${ }^{2}$

Although the detection and management of endoleaks after abdominal endovascular aortic aneurysm repair have been well described, few reports have been published about endoleaks after TEVAR. ${ }^{3-6}$ This investigation was performed to evaluate the incidence and the determinants of

\footnotetext{
From the Department of Surgical Sciences, Vascular Surgery Unit, ${ }^{\text {a }}$ Department of Surgical Sciences, ${ }^{\mathrm{b}}$ Cardiac Surgery Unit, Department of Radiology, ${ }^{\mathrm{c}}$ and Anaesthesia and Palliative Care, ${ }^{\mathrm{d}}$ Varese University Hospital, Varese, Italy; and Department of Cardiac Surgery, ${ }^{\mathrm{e}} 1$ st Chair of Cardiology and Cardiac Surgery, Medical University of Lodz, Lodz, Poland.

Received for publication July 10, 2008; revisions received Dec 5, 2008; accepted for publication Feb 3, 2009; available ahead of print April 10, 2009.

Address for reprints: Giovanni Mariscalco, MD, PhD, Department of Surgical Sciences, Cardiac Surgery Unit, Varese University Hospital, University of Insubria,

Viale Guicciardini 9, 21100 Varese, Italy (E-mail: giovannimariscalco@yahoo.it). J Thorac Cardiovasc Surg 2009;138:880-5

$0022-5223 / \$ 36.00$

Copyright (c) 2009 by The American Association for Thoracic Surgery doi:10.1016/j.jtcvs.2009.02.024
}

endoleaks after TEVAR. The outcomes of secondary interventions in patients with endoleaks were also evaluated.

\section{METHODS \\ Population and Management}

Between January 2000 and March 2008, 61 patients had endovascular repair for thoracic aortic aneurysm (TAA). Thoracoabdominal aneurysms and acute type B dissections were not considered in this analysis. TAAs included atherosclerotic or dissecting aneurysms $(n=52$, ruptured $n=4)$ and traumatic aneurysms $(n=9$, chronic $n=2)$. The study cohort contained 54 men, with a mean age of $63.6 \pm 17.9$ years (range: $17-87$ years). All elective patients had preoperative evaluation with echocardiography and spirometry; computed-tomography angiography (CT-A) of the brain was performed when the lesion involved the aortic arch or the distal portion of the arch in order to assess the integrity of the circle of Willis and the dominance of the vertebral arteries. Three different devices were used: Talent (Medtronic Vascular, Santa Rosa, Calif), Excluder (W. L. Gore and Associates, Flagstaff, Ariz), and TX-1/TX-2 (Cook, Bloomington, Ind). The type of SG has been chosen according to the aneurysm anatomic characteristics and type of lesion. Generally, self-expanding SGs without bare stents were used for traumatic aneurysms and proximal or distal extremity bare stents in the proximity of the epiaortic branches or abdominal visceral vessels.

The study protocol was in compliance with the local Institutional Review Board and received full approval. All patients gave their consent to participate.

\section{Follow-up and Imaging Characteristics}

After intervention, the follow-up imaging protocol included chest radiographs and triple-phase CT-A performed at 1, 4, and 12 months after SG 


$$
\begin{aligned}
& \text { Abbreviations and Acronyms } \\
& \begin{aligned}
\text { CI } & =\text { confidence interval } \\
\text { CT } & =\text { computed tomography } \\
\text { CT-A } & =\text { computed-tomography angiography } \\
\text { LSA } & =\text { left subclavian artery } \\
\text { OR } & =\text { odds ratio } \\
\text { SG } & =\text { stent graft } \\
\text { TAA } & =\text { thoracic aortic aneurysm } \\
\text { TEVAR } & =\text { endovascular repair of thoracic aortic } \\
& \text { aneurysm }
\end{aligned}
\end{aligned}
$$

implantation and annually thereafter. CT-A was performed using a 64-detector row (Aquilion, Toshiba, Zoetermeer, Netherlands); initially, nonenhanced computed tomography (CT) images were obtained, then 80 to 90 $\mathrm{mL}(350 / 400 \mathrm{mg}$ of iodine $/ \mathrm{mL}$ ) of a nonionic intravenous contrast material (Iomeron, Bracco, Milano, Italy) were administered at a rate of $4 \mathrm{~mL} / \mathrm{s}$ and followed by a bolus of $40 \mathrm{~mL}$ of saline solution by using a power injector (Envision CT Injector, Medrad, Pittsburgh, Pa). Arterial and venous phase acquisitions were then performed. Arterial phase imaging was performed by using bolus tracking. Arterial phase $\mathrm{CT}$ data acquisition was initiated when the attenuation of a region of interest positioned in the ascending aorta reached +150 Hounsfield units. Venous phase acquisition was initiated 90 seconds after the arterial phase. The nonenhanced CT arterial and venous phase acquisitions were initiated at the level of the upper portion of the neck and continued to the level of the celiac trunk. For nonenhanced CT arterial and venous phase acquisitions, a $0.50-\mathrm{mm}$ detector configuration was used. Each phase data acquisition was performed in less than 10 seconds. The postprocessing (multiplanar reconstructions, maximum intensity projection, 3-D images, and virtual angioscopy) was performed on a workstation (Vitrea, Vital Images, Plymouth, Minn). The patients were evaluated for the presence of an endoleak, type of sac reperfusion, aneurysm expansion, and endoleak intervention. Endoleaks were defined by a specific team of radiologists blinded to the performed procedures. The CT-A examinations were subsequently reevaluated on workstations by a team of a vascular surgeon and an interventional radiologist, using multiplanar reformatting capabilities and MIP/MPR/3-D reconstruction to identify and classify the type of endoleak.

\section{Definition}

The intervention was classified as emergency when surgery was performed within the first 24 hours after admission. ${ }^{7}$ The elective procedures performed on the same admission day were not considered emergent. Primary technical success was defined as successful deployment of the SG without any type of endoleak at the end of the intervention, and secondary technical success was defined as the persistent exclusion of the aortic disease after the second intervention (either endovascular or hybrid repair) without occurrence of any other type of endoleak. A hybrid repair, as well as "debranching," was intended as a combination of surgical and endovascular procedure. Location of the aortic disease was defined according to the classification proposed by Criado and colleagues ${ }^{8}$ in terms of landing zones. Type 1 endoleaks were classified on the basis of the location in contiguity with the proximal (type A) or distal (type B) attachment site. Endoleaks were classified as type 2 endoleak if the endoleak sac could not be seen communicating with the distal or proximal attachment site or if there was delayed enhancement of the endoleak sac. Type 3 endoleak was defined by the junctional separation of two SGs. Grading of the aortic arch atheroma was defined according to a previously reported modified classification ${ }^{9}$ : grade I (normal), smooth and continuous aortic intimal surface; grade II, intimal thickening 3 to $5 \mathrm{~mm}$; grade III, atheroma protruding $<5 \mathrm{~mm}$ into aortic lumen; grade IV, atheroma protruding $>5 \mathrm{~mm}$ into aortic lumen, and ulcerated or pedunculated. During the follow-up, period shrinking was defined a size reduction of $5 \mathrm{~mm}$ between 2 consecutive radiologic controls. For all patients, the mean changes in maximal aortic diameter were calculated by comparing the baseline aortic diameter with the maximal diameter at last follow-up, irrespective of endoleak treatment.

\section{Data Analysis}

Clinical data were prospectively recorded and tabulated with Microsoft Excel (Microsoft Corp, Redmond, Wash). Continuous variables were tested for normal distribution by the Kolmogorov-Smirnov test and compared between groups with unpaired Student $t$ test for normally distributed values; otherwise, the Mann-Whitney $U$ test was employed. In case of dichotomous variables, group differences were examined by chi-square or Fisher exact tests as appropriate.

A stepwise logistic regression model was developed to identify patient and procedural variables associated with endoleak development. The model was built using variables that demonstrated a $P$ value $<.20$ in univariable analysis. The strength of the association of variables with the endoleak was estimated by calculating the odds ratio (OR) and $95 \%$ confidence interval $(\mathrm{CI})$. The discrimination of the model was obtained by calculating the area under the receiver operating characteristic curve; the calibration of the model, by the Hosmer-Lemeshow goodness-of-fit test. Survival rate and freedom from reinterventions were estimated by means of the Kaplan-Meier method.

Results are expressed as mean \pm standard deviation for continuous variables and frequencies for the categorical ones. Statistical analysis was computed with SPSS, release 13.0 for Windows (SPSS Inc, Chicago, Ill).

\section{RESULTS}

\section{Clinical Univariable Data}

Among the 61 TEVAR cases, emergency procedures were performed in 20 patients $(31.7 \%)$. Endoleaks were detected in $9(14.5 \%)$ subjects, of which $7(77.8 \%)$ were type 1 (4 type A, 3 type B). None of the patients had more than 1 endoleak type. Endovascular or hybrid intervention was used to treat the endoleaks in all but 1 patient; 1 type $1 \mathrm{~A}$ endoleak spontaneously thrombosed. Five endoleaks (type 1A, $\mathrm{n}=4$ and type $2, \mathrm{n}=1$ ) were diagnosed during the initial postoperative CT-A at 30 days. Four endoleaks developed late; mean delay of endoleak appearance was 12 months (median: 9, range: 6-24 months). Patients with endoleak and patients without it were comparable in terms of clinical characteristics (Table 1). Briefly, age distribution was similar (72.2 \pm 5.1 vs $62.1 \pm 19.0$ years, $P=.176)$ as well as mean standard EuroSCORE (European System for Cardiac Operative Risk Evaluation) $(8.4 \pm 2.2$ vs $8.8 \pm 3.7, P=.751)$. No statistical differences were noted in terms of distribution of the aortic diseases. Four patients $(44.4 \%)$ in the endoleak group and $10(18.8 \%)$ among those without endoleak have had previous thoracic or abdominal aortic surgery.

\section{Morphological Univariable Data}

No differences were detected regarding the extension and morphology (saccular or fusiform) of the aneurysm $(P=$ .077 and $P=.478$, respectively). No differences were also observed regarding the surgical access and the SG used $(P=.796$ and $P=.724$, respectively). In contrast, the 
TABLE 1. Clinical characteristics of the enrolled patients

\begin{tabular}{lccc}
\hline \multicolumn{1}{c}{ Variable* } & $\begin{array}{c}\text { Endoleak } \\
(\mathbf{n}=\mathbf{9})\end{array}$ & $\begin{array}{c}\text { No endoleak } \\
(\mathbf{n = 5 2 )}\end{array}$ & $\boldsymbol{P}$ value \\
\hline Age & & & \\
$\quad$ Mean & $72.2 \pm 501$ & $62.1 \pm 19.0$ & .176 \\
$\quad$ Range & $64-80$ & $17-87$ & \\
Male sex, n (\%) & $8(88.9)$ & $46(88.5)$ & .999 \\
Hypertension, n (\%) & $9(100)$ & $40(76.9)$ & .184 \\
Diabetes, n (\%) & $0(0)$ & $4(7.7)$ & .999 \\
Dyslipidemia, n (\%) & $2(22.2)$ & $10(19.1)$ & .999 \\
COPD, n (\%) & $5(55.6)$ & $21(40.4)$ & .477 \\
Renal failure, n (\%) & $2(22.2)$ & $11(21.1)$ & .999 \\
Coronary artery disease, n (\%) & $1(11.1)$ & $15(28.8)$ & .423 \\
Cerebrovascular accident, n $(\%)$ & $1(11.1)$ & $7(13.5)$ & .999 \\
History of aneurysm, n $(\%)$ & $2(22.2)$ & $23(44.2)$ & .286 \\
EuroSCORE & $8.4 \pm 2.2$ & $8.8 . \pm 3.7$ & .751 \\
\hline COPD, Che
\end{tabular}

COPD, Chronic obstructive pulmonary disease; EruoSCORE, European System for Cardiac Operative Risk Evaluation. *For continuous variables: mean and standard deviation; for categorical variables: $\mathrm{n}(\%)$.

diameter of aneurysmal aorta between the 2 groups was significantly different $(P=.003$; Table 2$)$. SG diameter, length of covered aorta, and number of SGs were also significant $(P$ $=.003, P=.022, P=.002$, respectively). Lesions involving the proximal descending aorta were associated with lower endoleak occurrence $(11.1 \%$ vs $51.9 \%, P<.001)$. The left subclavian artery (LSA) was covered by SG in 24 cases $(88.9 \%$ vs $31.4 \%, P=.002)$.

\section{Outcome of Univariable Data}

Hospital mortality was $8.2 \%$; no difference was noted $(11.1 \%$ vs $7.7 \%, P=.563)$. The cause of death of the only patient who died in the endoleak group was rupture of an undetected type 1A endoleak that occurred 6 days after the intervention. The patient died while entering the operating room for an endovascular attempt to seal the rupture. Conversely, in the control group, 2 patients died because of a myocardial infarction and 2 of respiratory failure. Postoperative complications were registered in 12 patients. Cerebrovascular accidents were observed in $6(9.8 \%)$ subjects. In detail, 3 patients had transient ischemic attack without sequelae, and the other 3 subjects had major cerebrovascular events (stroke). Paraplegia was not encountered in our population. Concerning renal failure, 4 patients $(4.2 \%)$ had a transient increase in creatinine level $(>200 \mu \mathrm{mol} / \mathrm{L})$, whereas 2 subjects required temporary dialysis therapy for a median of 48 hours.

No open conversions were performed; primary technical success rate was $95 \%$ (58 of 61 cases). In the endoleak group, 5 patients required additional vascular or endovascular procedures to complete or in association with TEVAR (55.6\% vs $36.5 \%, P=.298)$. Secondary technical success rate was $100 \%$; intervention included additional SG $(\mathrm{n}=4)$ and total debranching $(\mathrm{n}=2)$ to treat type 1 endoleaks, whereas both type 2 endoleaks were sealed with embolization of the origin
TABLE 2. Aneurysm and procedure details

\begin{tabular}{|c|c|c|c|}
\hline Variable* & $\begin{array}{c}\text { Endoleak } \\
(\mathbf{n}=9)\end{array}$ & $\begin{array}{l}\text { No endoleak } \\
(\mathrm{n}=\mathbf{5 2}) \\
\end{array}$ & $P$ value \\
\hline \multicolumn{4}{|l|}{ TAA characteristics } \\
\hline Diameter (mm) & $7.7 \pm 2.2$ & $5.4 \pm 1.6$ & .033 \\
\hline Length (mm) & $16.0 \pm 8.1$ & $11.7 \pm 7.8$ & .077 \\
\hline TAA morphology & & & .478 \\
\hline Saccular, n (\%) & $3(33.3)$ & $26(50.0)$ & \\
\hline Fusiform, n (\%) & $6(66.7)$ & $26(50.0)$ & \\
\hline Aortic atheroma degree & & & .843 \\
\hline Grade I & $4(44.4)$ & $29(55.8)$ & \\
\hline Grade II & $4(44.4)$ & $16(30.8)$ & \\
\hline Grade III & $1(11.1)$ & $6(11.5)$ & \\
\hline Grade IV & $0(0)$ & $1(1.9)$ & \\
\hline \multicolumn{4}{|l|}{ SG characteristics } \\
\hline Stent diameter $(\mathrm{mm})$ & $39.0 \pm 2.5$ & $34.8 \pm 4.3$ & .003 \\
\hline Coverage length (mm) & $20.7 \pm 5.8$ & $18.3 \pm 20.3$ & .022 \\
\hline No. stents & $1.9 \pm 0.9$ & $1.2 \pm 0.5$ & .002 \\
\hline SG landing zone & & & $<.001$ \\
\hline LCA, n (\%) & $3(33.6)$ & $2(3.8)$ & \\
\hline LSA, n (\%) & $5(55.6)$ & $7(13.5)$ & \\
\hline Prox DTA, n (\%) & $1(11.1)$ & $27(51.9)$ & \\
\hline Dist DTA, n (\%) & $0(0)$ & $16(30.8)$ & \\
\hline SG used & & & .724 \\
\hline Excluder, n (\%) & $4(44.4)$ & $27(51.9)$ & \\
\hline Talent, n (\%) & $3(33.3)$ & $11(21.2)$ & \\
\hline TX-1/TX-2, n (\%) & $2(22.2)$ & $4(26.9)$ & \\
\hline LSA coverage & $8(88.9)$ & $16(31.4)$ & .002 \\
\hline Surgical access & & & .796 \\
\hline Femoral artery & $8(88.9)$ & $46(88.5)$ & \\
\hline Iliac artery & $1(11.1)$ & $4(7.7)$ & \\
\hline Aorta & $0(0)$ & $2(3.8)$ & \\
\hline
\end{tabular}

$L C A$, left carotid artery; $L S A$, left subclavian artery; $D T A$, descending thoracic aorta; $S G$, stent graft; TAA, thoracic aortic aneurysm; Prox, prosimal; DIST, distal. *For continuous variables: mean and standard deviation; for categorical variables: $\mathrm{n}(\%)$.

of the LSA. Clinical and imaging follow-up was $100 \%$ complete as no surviving patient was lost to follow-up. Mean follow-up was 32.4 months (median: 26, range: 1-96 months). The percentage of patients free from reintervention was $94.6 \% \pm 3.0 \%, 88.3 \% \pm 4.5 \%$, and $85.4 \% \pm 5.2 \%$, at 1,2 and 5 years, respectively (Figure 1, $A$ ). The actuarial survival estimates at 1,2 , and 5 years after the procedure were $85.2 \% \pm 4.6 \%, 78.1 \% \pm 5.4 \%$, and $70.6 \% \pm$ $6.4 \%$, respectively (Figure $1, B$ ). Follow-up mortality occurred in 12 patients, including 3 with respiratory failure, 6 with myocardial infarction, 2 with lung cancer, and 1 with massive pulmonary embolism.

Two patients developed further endoleaks during the follow-up period: 1 patient treated for a type 1B endoleak developed a type 2 from the intercostal-thyroid trunk that was embolized, and 1 patient treated for a type 2 endoleak developed a type $1 \mathrm{~A}$ endoleak that required total debranching.

\section{Multivariable Analysis}

Multivariate analysis demonstrated that the diameter of aneurysmal aorta (OR 1.75, 95\% CI 1.07-2.86) and the 

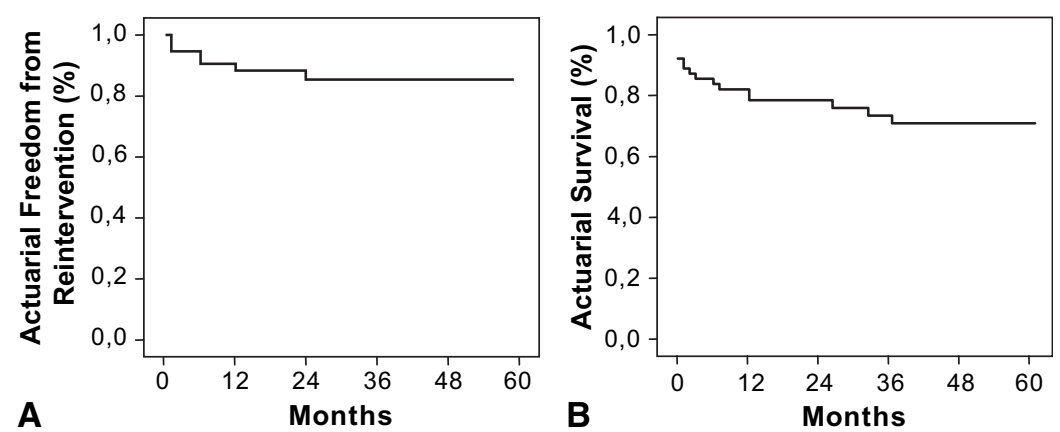

FIGURE 1. Actuarial freedom form reintervention (A) and actuarial survival (B).

coverage of the LSA (OR 12.05, 95 CI\% 1.28-113.30) were independently associated with endoleak development. With all patients considered, the variables of the model correctly predicted $87 \%$ of the observed cases of postoperative endoleak. The area under the receiver operating characteristic curve for this model was .896, suggesting a good ability to distinguish between individuals who will develop endoleak and those who will not. The Hosmer-Lemeshow goodness-of-fit test was not significant for lack of fit $\left(\chi^{2}[7 \mathrm{df}]=2.79, P=.904\right)$, indicating that there was no statistically significant departure from a perfect fit. Figure 2 displays the probability of patient endoleak occurrence in relation to the number of $\mathrm{SG}$, native aortic diameter, and LSA coverage.

\section{DISCUSSION}

In contrast to the available data on endoleaks after abdominal aortic repair, little has been published regarding the incidence and predictive factors of endoleak after TEVAR. ${ }^{4,10}$ Earlier series reported an endoleak rate in the range of $5 \%$ to $30 \%$, including data from single-center series and multicenter registries. ${ }^{4-6,10,11}$ In the interesting analysis of Parmer and colleagues, ${ }^{4}$ the authors published a $29 \%$ endoleak rate in a series of 105 patients treated for TAAs only. Our experience is somewhat different; in fact, we observed a lower endoleak occurrence $(14.7 \%)$ without differences in terms of comorbidities or disease characteristics between patients with and without endoleaks. In our patient population, a larger aortic diameter at the time of repair was predictive of endoleaks, but in contrast to the data of Parmer and associates ${ }^{4}$ the disease extension was not statistically predictive of postoperative endoleak occurrence. Moreover, a higher number of devices and a larger size did not provide superior results in terms of endoleak prevention.

In most of the published studies, endoleaks were related to the proximal or distal landing zones $(12,13)$. Verhoye and colleagues, ${ }^{10}$ in a retrospective analysis of 54 TEVAR procedures, observed a relevant endoleak rate of $40 \%(9 /$ 22 cases, 1 ruptured) for degenerative TAAs and concluded that longer SG and a longer $(>3 \mathrm{~cm})$ neck reduced the likelihood of proximal endoleaks. In our series, a lengthier aortic coverage was not protective against endoleaks, suggesting that a more liberal stenting of normal aorta is not justified. Conversely, the proximal landing zone strongly predicted a TEVAR failure, especially when SG attachment involved the proximity of the LSA, which resulted in an independent risk for postoperative endoleaks. Our findings are supported by the outstanding study of Demers and colleagues ${ }^{12}$ : in their series, the transposition of the LSA was an independent risk factor for treatment failure, probably reflecting a suboptimal proximal landing zone because of pathological anatomy. Moreover, abrupt angulation, vector forces acting on the SG, and cantilevering the proximal SG end off the lesser curve of the distal arch have also been implicated in endoleak formation. ${ }^{12}$ Therefore, the proximal landing zone in the rectilinear portion of the descending aorta should be strongly regarded as the most favorable target to obtain an effective and persistent sealing. Nevertheless, overstenting

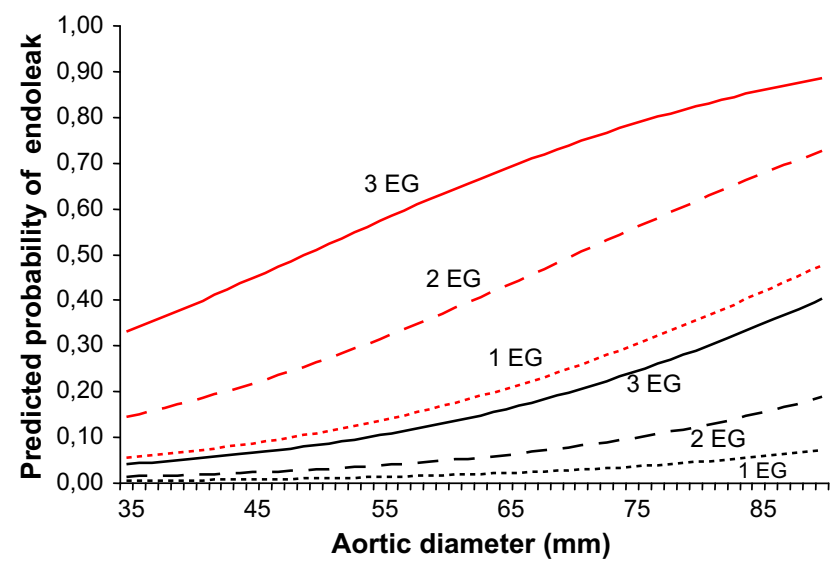

FIGURE 2. Predicted probability for developing endoleak by the diameter of the aneurysmal aorta $(\mathrm{mm})$ and the number of the inserted stent grafts with reference to the position of the landing zone (red line, coverage of left subclavian artery; black lines, no coverage of left subclavian artery). As the diameter of the aneurysmal aorta and number of stent grafts increase with coverage of the left subclavian artery, the probability of developing endoleak also increases (eg, for a patient with $60 \mathrm{~mm}$ of native aorta diameter requiring 3 stent grafts and the coverage of left subclavian artery, the probability of developing an endoleak is about $65 \%$ ). 
of the LSA with or without its selective revascularization or total epiaortic debranching should be more frequently performed to avoid an increased risk of endoleaks formation. Tailoring an appropriate landing zone remains mandatory to obtain efficacious immediate and durable results.

The longer duration of follow-up reported herein allowed the detection of several late complications. Demers and associates $^{12}$ showed actuarial freedom from primary or secondary endoleak of $78 \%$ and $64 \%$ at 1 and 5 years, respectively, and actuarial freedom from aortic reintervention was $83 \%$ and $77 \%$ at these same times. Similar freedom from reintervention was observed in our patients. Concerning the rate of surgical conversion, our data are in accordance with Grabenwoger and colleagues, ${ }^{6}$ who reported a $5 \%$ rate of surgical conversion after TEVAR. A higher percentage of reintervention after SG treatment for atherosclerotic aneurysms was also noted in our patients. We demonstrated that the chronic enlargement of the noncovered aorta favored the development of type 1 endoleaks. However, we did not confirm that a shorter landing zone is a predictive factor for endoleaks.

Secondary type 1 endoleaks have been thought to be also the result of SG migration following inadequate fixation. ${ }^{14}$ According to the literature, late migration occurred with an incidence of $0 \%$ to $30 \%$, typically higher for early generation devices. ${ }^{8,13,15}$ Like Demers and colleagues, ${ }^{12}$ we did not observe SG migration during follow-up in patients with secondary endoleaks. However, this complication should be promptly suspected and then recognized with early reexamination. ${ }^{4,10,16}$ In our experience, this recommendation is supported by the fact that the only patient who died of a procedure-related complication in the endoleak group had a fatal rupture of the aneurysmal sac after an undetected endoleak. Type 1 endoleak should be treated aggressively when found. Similar to the approach suggested for endoleak after abdominal endovascular repair, thoracic endoleaks have been suggested to be preferentially approached with an endovascular procedure. ${ }^{17,18}$ Our experience is consistent with the findings of Verhoye and colleagues, ${ }^{10}$ who surgically converted just 1 of the 16 endoleaks detected postoperatively. Satisfactory repair of all endoleaks in our study was confirmed by the $100 \%$ secondary technical success leading to persistent exclusion of the aortic disease.

This study is limited by its interpretations from a single center only and further, by its retrospective analysis, although data were prospectively collected. However, the study design is shared with previous reports. ${ }^{3-6}$ The study is also limited in statistical terms based on relatively few observations. Another limitation of the study was the lack of information concerning the aortic diameter at the landing zones and the length of landing zones. However, these variables are hard to define, and the measurements of the thoracic aorta, especially in the arch curve, are very difficult to standardize; in addition, the proximal sealing point is not so exactly respected during the deployment procedure as well as the length of landing zones.

In conclusion, in our study, the diameter of the aneurysmal aorta and the position of the landing zone are the most relevant factors in endoleak occurrence after TEVAR procedures. A careful follow-up program should be mandatory, because most endoleaks may be treated successfully by additional endovascular procedures. Moreover, overstenting the LSA with a subsequent LSA revascularization when required or the entire epiaortic debranching should be more frequently performed to prevent endoleak formation.

\section{References}

1. Schepens MA, Kelder JC, Morshuis WJ, Heijmen RH, van Dongen EP, ter Beek HT. Long-term follow-up after thoracoabdominal aortic aneurysm repair. Ann Thorac Surg. 2007;83:S851-5.

2. Quinones-Baldrich WJ. Descending thoracic and thoracoabdominal aortic aneurysm repair: 15-year results using a uniform approach. Ann Vasc Surg. 2004; 18:335-42.

3. Prinssen M, Verhoeven E, Buth J, Cuypers PW, van Sambeek MR, Balm R, et al. A randomized trial comparing conventional and endovascular repair of abdominal aortic aneurysms. N Engl J Med. 2004;351:1677-9.

4. Parmer SS, Carpenter JP, Stavropoulos SW, Fairman RM, Pochettino A, Woo EY, et al. Endoleaks after endovascular repair of thoracic aortic aneurysms. $J$ Vasc Surg. 2006;44:447-52.

5. Leurs L, Bell R, Degrieck Y, Thomas S, Hobo R, Lundbom J. Endovascular treatment of thoracic aortic diseases: combined experience from the EUROSTAR and United Kingdom Thoracic Endograft registries. J Vasc Surg. 2004;40:670-80.

6. Grabenwoger M, Fleck T, Ehrlich M, Czerny M, Hutschala D, Schoder M, et al. Secondary surgical interventions after endovascular stent-grafting of the thoracic aorta. Eur J Cardiothorac Surg. 2004;26:608-13.

7. Roques F, Nashef SA, Michel P, Gauducheau E, de Vincentiis C, Baudet E, et al. Risk factors and outcome in European cardiac surgery: analysis of the EuroSCORE multinational database of 19030 patients. Eur J Cardiothorac Surg. 1999;15:816-22.

8. Criado FJ, Clark NS, Barnatan MF. Stent graft repair in the aortic arch and descending thoracic aorta: a 4-year experience. J Vasc Surg. 2002;36:1121-8.

9. Gutsche JT, Cheung AT, McGarvey ML, Moser WG, Szeto W, Carpenter JP, et al. Risk factors for perioperative stroke after thoracic endovascular aortic repair. Ann Thorac Surg. 2007;84:1195-200.

10. Verhoye JP, de Latour B, Heautot JF, Vola M, Langanay T, Corbineau H, et al. Mid-term results of endovascular treatment for descending thoracic aorta diseases in high-surgical risk patients. Ann Vasc Surg. 2006;20:714-22.

11. Riesenman PJ, Farber MA, Mendes RR, Marston WA, Fulton JJ, Mauro M, et al. Endovascular repair of lesions involving the descending thoracic aorta. $J$ Vasc Surg. 2005;42:1063-74.

12. Demers P, Miller DC, Mitchell RS, Kee ST, Sze D, Razavi MK, et al. Midterm results of endovascular repair of descending thoracic aortic aneurysms with first-generation stent grafts. J Thorac Cardiovasc Surg. 2004;127:664-73.

13. Alric P, Hinchliffe RJ, Wenham PW, Whitaker SC, Chuter TAM, Hopkinson BR. Lessons learned from the long-term follow-up of a first-generation aortic stentgraft. J Vasc Surg. 2003;37:367-73.

14. Hansen C, Bui H, Donayre C, Aziz I, Kim B, Kopchok G, et al. Complications of endovascular repair of high-risk and emergent descending thoracic aortic aneurysms and dissections. J Vasc Surg. 2004;40:228-34.

15. Czerny M, Cejna M, Hutschala D, Fleck T, Holzenbein T, Schoder M, et al. Stentgraft placement in atherosclerotic decending thoracic aneurysms: midterm results. J Endovasc Ther. 2004;11:26-32.

16. Stavropoulos $S$, Baum R. Imaging modalities for the detection and management of endoleaks. Semin Vasc Surg. 2004;17:154-60.

17. Sampaio SM, Panneton JM, Mozes GI, Andrews JC, Bower TC, Karla M, et al. Proximal type I endoleak after endovascular abdominal aortic aneurysm repair: predictive factors. Ann Vasc Surg. 2004;18:621-8.

18. Erer D, Banach M, Ugurlucan M. Endovascular treatment of mycotic aortic aneurysms. J Thorac Cardiovasc Surg. 2008;135:1404-5. 


\section{Appendix. Variables Included in Multivariable Analysis}

Clinical variables: age, hypertension, length of stay, bleeding (12 hours).

Operative variables: thoracic aortic aneurysm length, thoracic aortic aneurysm diameter, stent graft diameter, stent graft coverage length, number of stent grafts, stent graft landing zone, left subclavian artery coverage. 